DISTRIBUTION STATEMENT A. Approved for public release; distribution is unlimited.

\title{
Environmental Acoustics and Intensity Vector Acoustics with Emphasis on Shallow Water Effects and the Sea Surface
}

\author{
Peter H. Dahl \\ Applied Physics Laboratory \\ University of Washington \\ Seattle, Washington 98105 \\ phone: (206) 543-2667 fax: (206) 543-6785 email:dahl@apl.washington.edu
}

Award Number: N00014-04-1-0111

\section{LONG-TERM GOALS}

To understand and predict key properties of the signal intensity vector field as it propagates away from an active sound source, with emphasis is on mid-frequency, shallow water propagation. New and enabling technologies such as MEMS and bio-inspired transduction, will allow tomorrow's Navy to exploit acoustic vector field properties (velocity, acceleration, intensity) much more than today's. Furthermore, advancement of current Navy capabilities will be in part realized through a better understanding of the environmental and acquisition geometry dependence (source depth, range, etc.) of the vector field in a shallow water environment.

\section{OBJECTIVES}

The primary technical objectives this year involve our measurements from TREX13 conducted in spring 2013, directed towards our participation at the TREX13 workshops in San Francisco (December 2013) and Indianapolis (October 2014). These objectives are: (1) analysis of directional wave spectra and associated synthetic sea surfaces for the TREX13 research teams to model reverberation and time spreading in propagation, (2) estimate the bottom reflection coefficient using both a conventional technique (energy versus arrival angle) and with a new technique based vector acoustics, and (3) estimate and construct intensity streamlines that be used to better understand the forward propagating field.

These objectives share a common thread: towards understanding the environmental influences on the intensity vector field in shallow water sound propagation and reverberation.

\section{APPROACH}

For 2013 TREX13 work, we incorporated a field measurement geometry (Fig. 1) in our experimental design that was motivated by the above-mentioned common thread. More specifically, directional wave measurements were made using one wave buoy at the TREX13 reverberation source location (near the research vessel R/V Sharp) and one buoy located $5 \mathrm{~km}$ distant along bearing $129^{\circ}$ (near the research vessel R/V Smith).

Bearing $129^{\circ}$ constituted the primary look angle for the reverberation measurements; our team made related propagation measurements both parallel, and perpendicular to this primary look angle to study the role of directional waves on both reverberation and related propagation. These measurements were recorded on 


\section{Report Documentation Page}

Form Approved

OMB No. 0704-0188

Public reporting burden for the collection of information is estimated to average 1 hour per response, including the time for reviewing instructions, searching existing data sources, gathering and maintaining the data needed, and completing and reviewing the collection of information. Send comments regarding this burden estimate or any other aspect of this collection of information,

including suggestions for reducing this burden, to Washington Headquarters Services, Directorate for Information Operations and Reports, 1215 Jefferson Davis Highway, Suite 1204, Arlington

VA 22202-4302. Respondents should be aware that notwithstanding any other provision of law, no person shall be subject to a penalty for failing to comply with a collection of information if it

does not display a currently valid OMB control number.

1. REPORT DATE

30 SEP 2014

4. TITLE AND SUBTITLE

Environmental Acoustics and Intensity Vector Acoustics with Emphasis on Shallow Water Effects and the Sea Surface

6. AUTHOR(S)

7. PERFORMING ORGANIZATION NAME(S) AND ADDRESS(ES)

University of Washington,Applied Physics Laboratory,1013 NE 40th Street,Seattle,WA,98105

9. SPONSORING/MONITORING AGENCY NAME(S) AND ADDRESS(ES)

3. DATES COVERED

00-00-2014 to 00-00-2014

5a. CONTRACT NUMBER

5b. GRANT NUMBER

5c. PROGRAM ELEMENT NUMBER

5d. PROJECT NUMBER

5e. TASK NUMBER

5f. WORK UNIT NUMBER

8. PERFORMING ORGANIZATION REPORT NUMBER

10. SPONSOR/MONITOR'S ACRONYM(S)

11. SPONSOR/MONITOR'S REPORT NUMBER(S)

12. DISTRIBUTION/AVAILABILITY STATEMENT

Approved for public release; distribution unlimited

13. SUPPLEMENTARY NOTES

14. ABSTRACT

15. SUBJECT TERMS

16. SECURITY CLASSIFICATION OF:

a. REPORT

unclassified b. ABSTRACT

unclassified c. THIS PAGE

unclassified
17. LIMITATION OF ABSTRACT

Same as

Report (SAR)
18. NUMBER 19a. NAME OF

OF PAGES RESPONSIBLE PERSON

8 
receiving station (known as MORAY) consisting of a combined pressure and vector sensor (4 channels), a 7 element vertical line array and a 4-elment horizontal line array of pressure sensors (Fig. 3.). The combined set of vector sensors and array elements established a set of 15 channels that were coherently recorded.

Key individuals are post-doctoral research associate David Dall’Osto (APL-UW), Jeewoong Choi (Hanyang University, Ansan Korea) who visited APL-UW from July 2013-June 2014 funded by ONR global, as well as other researchers involved in TREX13.



Figure 1. Right side: Basic geometry of the TREX experiment with focus on our measurements located $5 \mathrm{~km}$ from the $\mathrm{R} / \mathrm{V}$ Sharp. Two key bearings $129^{\circ}$ forming the primary look-angle for reverberation measurements using the sources co-located with the $R / V$ Sharp, and the perpendicular direction $219^{\circ}$. Left side: underwater photograph of the MORAY tower housing vertical and horizontal line arrays and vector sensor.

\section{WORK COMPLETED}

Our work this year on TREX data is organized into three areas concerning: (1) Directional Waves, (2) Comparative estimates of bottom loss using conventional (time and angle) technique and vector based technique, and (3) estimating energy flux streamlines in propagation measurements. Preliminary results were first discussed at the TREX workshop in December 2013 with subsequent results since this time to be discussed at the upcoming workshop in October 2014. A summary of this work is given in the next section. 


\section{RESULTS}

\section{Directional Waves}

The directional measurements made by our group is being used by multiple TREX13 research teams. For this, three distinct conditions have been identified (Fig. 2) representing very calm conditions (case 1), relatively windy conditions for which the wave field and wind direction are not closely aligned (case 2), and similar windy conditions for which the wind and wave directions show a high degree of alignment (case 3).
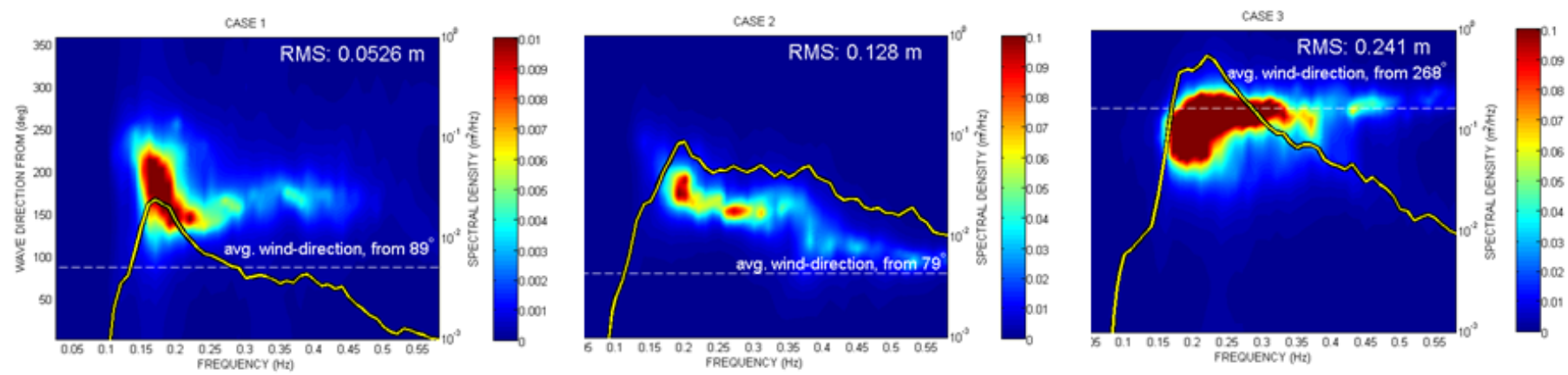

Figure 2. Three distinct conditions currently being investigated for environment effects on propagation and reverberation by multiple TREX13 research teams. Case 1: very calm conditions wind speed $\sim 3 \mathrm{~m} / \mathrm{s}$, rms waveheight $\sim 0.05 \mathrm{~m}$. Cases 2 and 3: higher seas state conditions with speed $\sim 8 \mathrm{~m} / \mathrm{s}$ each case and differing waveheights, 0.13 and $0.24 \mathrm{~m}$, owing to differing wind directions.

The corresponding 1-D folded wavenumber spectra used for input to propagation and reverberation modeling are shown in Fig. 3. These spectra depict sea surface roughness as a function of length scale or wavenumber, along bearing $129^{\circ}\left(\mathrm{k}_{1}\right)$ and the direction perpendicular to this bearing $219^{\circ}\left(\mathrm{k}_{2}\right)$. (Interpreting $\mathrm{F}\left(\mathrm{k}_{1}, \mathrm{k}_{2}\right)$ as a probability density function of roughness scale, then a folded spectrum is the marginal density generated by way of separate integration of $\mathrm{F}\left(\mathrm{k}_{1}, \mathrm{k}_{2}\right)$ with respect to $\mathrm{k}_{1}$ and $\mathrm{k}_{2}$, with folding representing a combining of resulting negative and positive wavenumbers.) Of particular interest is case 2 where the two arrows identify a reversal in the properties of shorter and longer length scales along the two propagation directions.

Results such as those shown in Fig. 3 have been provided to TREX13 researchers using scattering approaches to model directional sea surface effects on reverberation. For other research teams, e.g, TNO, The Hague, The Netherlands, we have generated synthetic sea surfaces corresponding to a 1-D cut through a 2-D sea surface using the approach outlined in ref. [1]. These surfaces will be used to assess time spreading and other propagation effects using parabolic wave based simulations. 


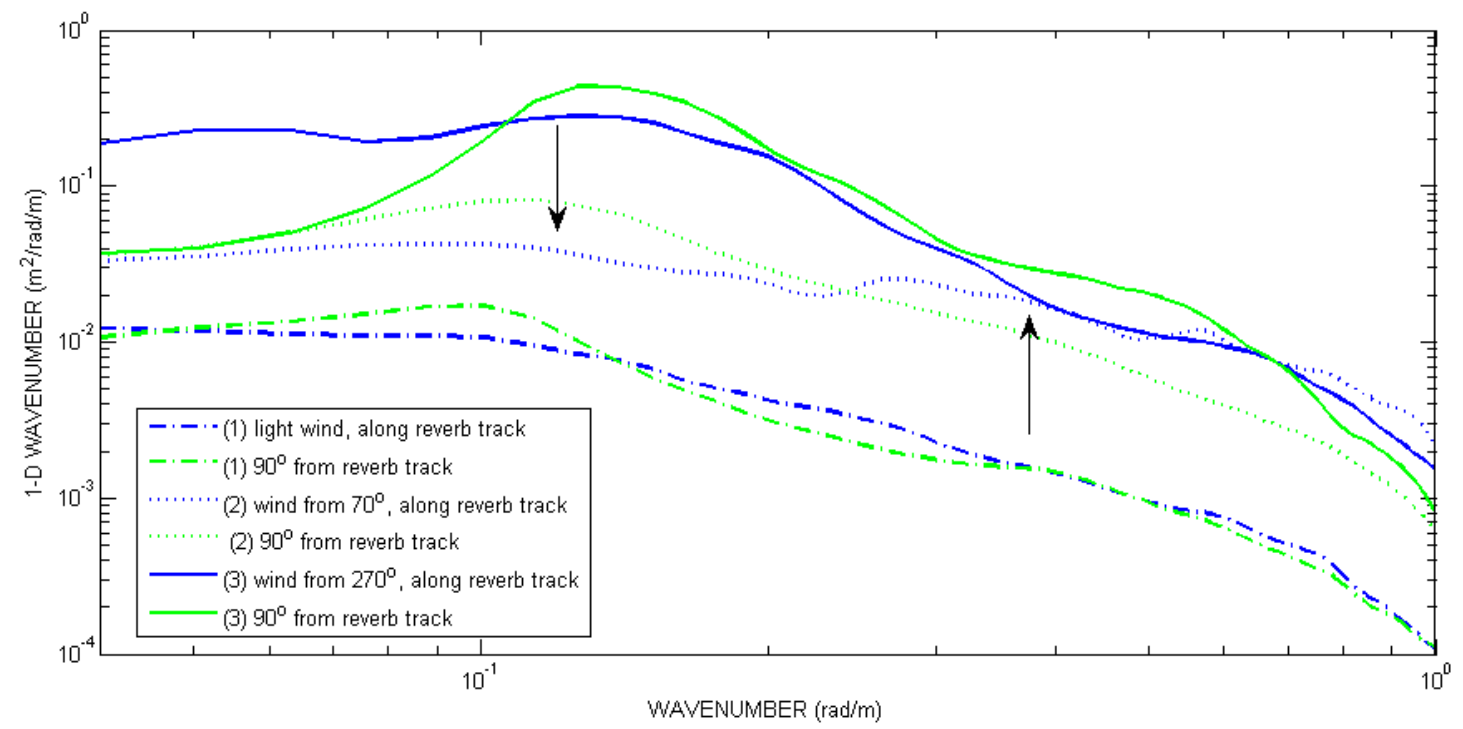

Figure 3. 1-D folded wavenumber spectra corresponding to the three cases shown in Fig. 2 used for input to propagation and reverberation modeling.

\section{Circularity and Geoacoustic Inversion}

The degree of circularity is a non-dimensional vector quantity relating to acoustic particle velocity [2]. For example, if two wavefronts are in phase, acoustic particle motion falls along a line with an orientation related to the relative magnitude of the direct and reflected field. When the two wavefronts are out of phase, the motion follows a curved path which will be purely circular when the two wavefronts are of equal magnitude and 90 degrees out of phase.

This motion can be succinctly described by degree of circularity, which itself is a vector and defined as follows [2] ,

$$
\vec{\Theta}=\frac{\operatorname{Im}\left\{\overrightarrow{\mathbf{v}} \times \overrightarrow{\mathbf{v}}^{*}\right\}}{|\overrightarrow{\mathbf{v}}|^{2}}=2 \frac{\overrightarrow{\mathbf{Q}} \times \overrightarrow{\mathbf{I}}}{|p|^{2}|\overrightarrow{\mathbf{v}}|^{2}}
$$

where $\vec{\Theta}$ is dependent on the relative orientation of the active intensity, $\overrightarrow{\mathbf{I}}$, and reactive intensity, $\overrightarrow{\mathbf{Q}}$, vectors and is non-dimensionalized by normalizing by the magnitude of the pressure and particle velocity fields. The values of $\vec{\Theta}$ fall between $+/-1$ where 0 indicates a particle motion path falling entirely on a straight line and $+/-1$ indicates purely circular motion in a counter-clockwise or clockwise direction, respectively. In general, circular motion occurs in regions of destructive interference, while straight line motion occurs in regions of constructive interference.

In TREX13, $\vec{\Theta}$ was measured as a function of depth by gradually raising a source from a depth $12 \mathrm{~m}$ to approximately $1 \mathrm{~m}$ during which the source-receiver range was held stationary (50 to $75 \mathrm{~m}$ depending on test.) The result, Fig. 4, shows how $\vec{\Theta}$ varies with source depth which we subsequently interpret with both a simple model and with parabolic equation modeling, for which results are summarized in a manuscript in preparation (Dall’Osto, Choi and Dahl, “Multipath Interference Effects on Acoustic Particle Motion in Shallow Water, 
2014.) Of key interest is the apparent sensitivity of $\vec{\Theta}$ towards bottom geoacoustic parameters, with bottom sound speed of $1615 \mathrm{~m} / \mathrm{s}$ providing the best fit to the data and with variation of approximately $50 \mathrm{~m} / \mathrm{s}$ from this value resulting in clear mismatch with the data. This same manuscript also shows results from more conventional estimate of bottom loss (Fig. 5) using intensity versus arrival angle and based on the same data. These two estimates at $1 \mathrm{kHz}$ (circularity) and at $6 \mathrm{kHz}$ (conventional method) indicate frequency dependence in the bottom sound speed. The vector method, which does not require multipath separation, can be used to determine the sound speed as a function of frequency.

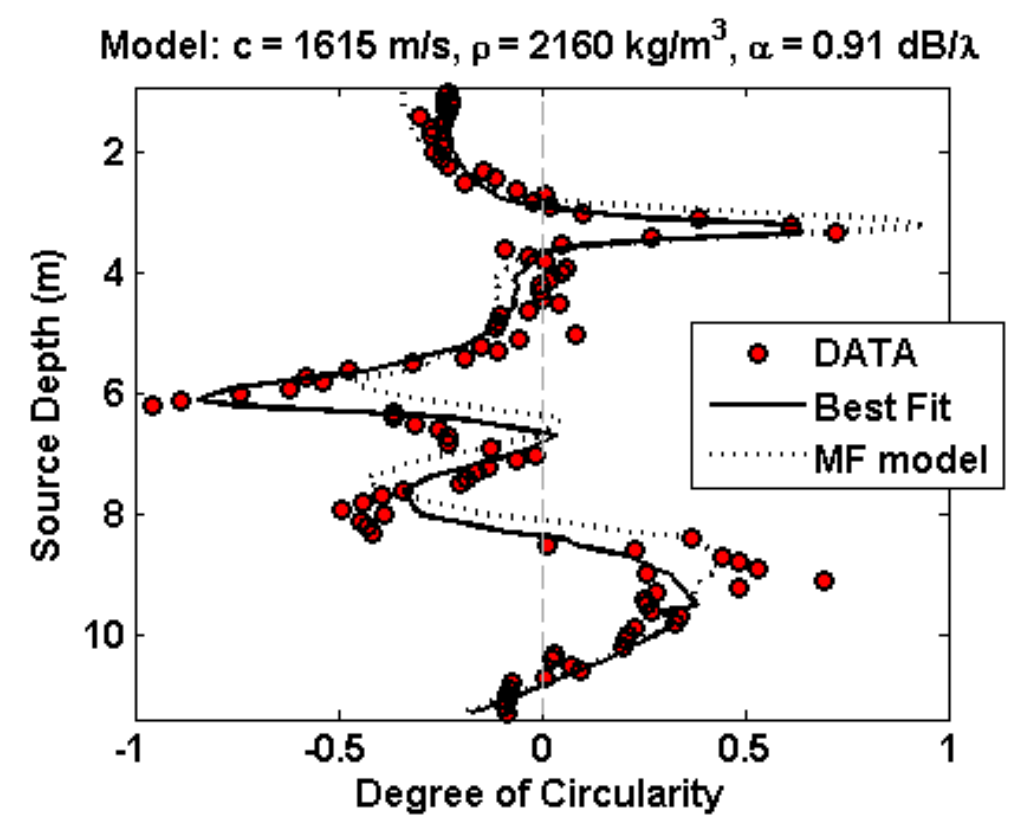

Figure 4. Measurements of degree of circularity $\vec{\Theta}$ versus source depth (red symbols) at $1025 \mathrm{~Hz}$ compared with a model based on the best fit of geoacoustic parameters and a model determined by conventional means at a higher frequency $(6 \mathrm{kHz})$.

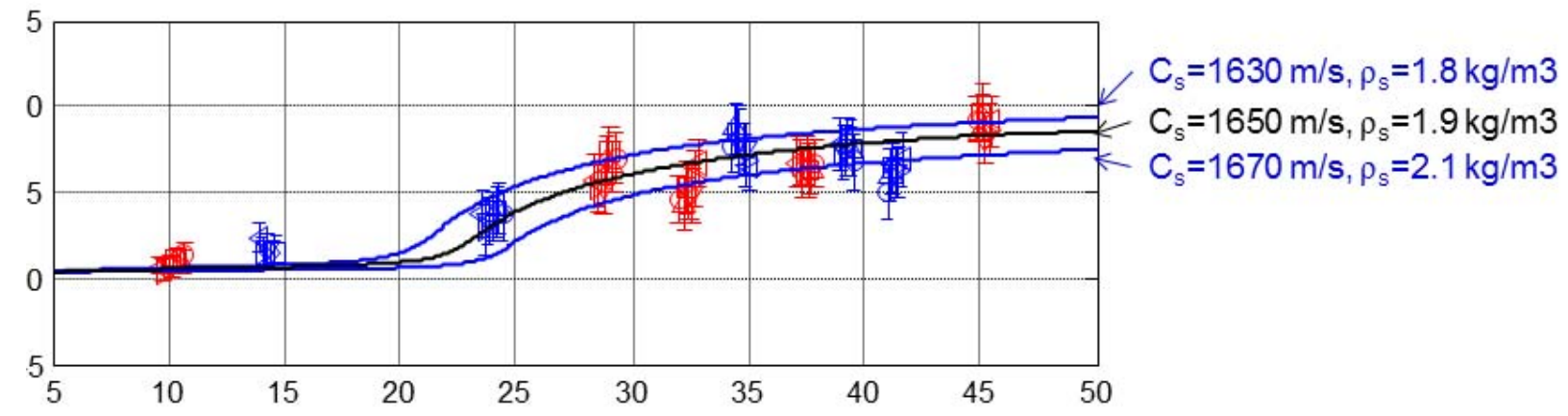

Figure 5. Direct measurements of the bottom reflection loss (blue and red data) versus models (solid lines) based geoacoustic parameters shown on right. 


\section{Intensity Streamlines}

Interference among modes or rays can establish a null, or dislocation, where the acoustic pressure field vanishes. Measurements of the acoustic vector field made during TREX13 are used to examine the active intensity vector field near such dislocations. The measurements were made during a drift experiment during which the research vessel, from which an acoustic source was suspended, slowly drifted away from the vector receiving array (Fig. 1) forming a synthetic horizontal aperture.

Energy flux streamlines drawn parallel to the active intensity vector field illustrate vortex structures such as those shown in Fig. 6 which are result of parabolic equation based modeling.



Figure 6. Numerically simulated active intensity vector field at $1025 \mathrm{~Hz}$ and accompanying streamlines (black lines) corresponding to a segment of the TREX13 drift experiment. The underlaying color map indicates the value of an impedance ratio.

Figure 7 depicts true, calibrated estimate of these streamlines including regions containing vortices, with details on Figs. 6 and 7 presented in the manuscript, Dall'Osto and Dahl, "Measurements of dislocations and intensity vortices”, J. Acoust. Soc. Am., 2014, which remains under review. 


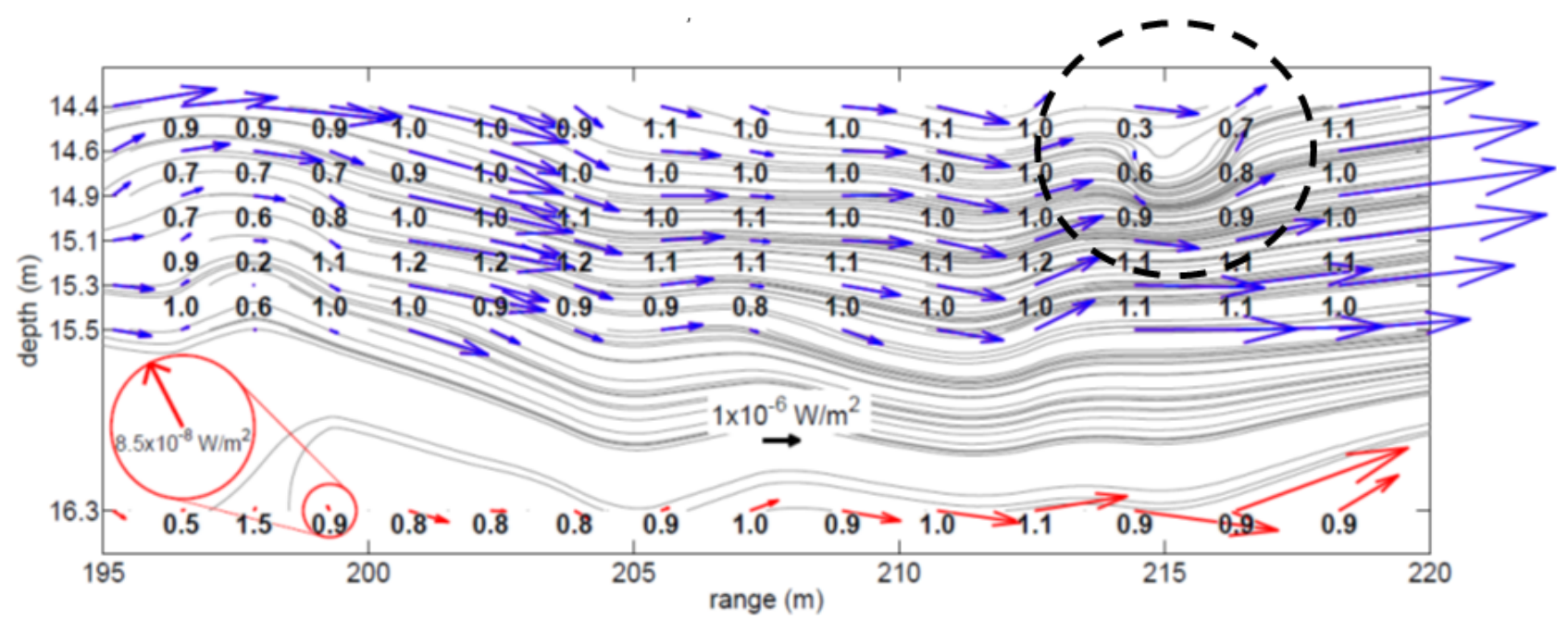

Figure 7. Active intensity vector field at $1025 \mathrm{~Hz}$, measured by the vector sensor (red arrows) and the VLA (blue arrows). The length of the arrows are proportional to the intensity magnitude. The red circle shows a magnification of a vector sensor measurement with horizontal intensity directed toward the source. Streamlines constructed from the measured active intensity are shown by the gray lines. Measurements of the impedance ratio are indicated by the numbers.

\section{IMPACT/APPLICATIONS}

Our work on the effects of the directional wave spectrum, e.g., as in [1], has shown how directional waves can have a direct impact on reverberation in shallow water. This has motivated the new measurements directional waves from TREX13 which are currently being used to more directly assess the influence of the sea surface on shallow water reverberation.

Our studies in vector acoustics and in particular vector circularity as summarized in [2] has led to potential applications involving the detection of sources in shallow water where match field approaches have performed poorly. Though a source detection application is yet fully realized, the measurements vector circularity from TREX13 (item 2 in RESULTS), are pointing to related geoacoustic application.

Our work on visualizing the acoustic vector field through intensity streamlines shows unique properties in the vector field near dislocations. In regions containing dislocations, the vector field is highly sensitive to the individual acoustics paths contributing to the complete destruction of the field. A change in one of these paths, such as the forward scattered field from a moving target, is readily observed in these vector properties. Similarly, the stability of vector properties near dislocations can be used to assess the effect the sea surface on the reverberation field.

\section{RELATED PROJECTS}

The PI is also advising PhD student Mr. Jeffrey Daniels, from the Acoustics Research Detachment (Bayview ID) Carderock Division, who has received an ILIR grant from ONR to study new vector sensing technologies at the University of Washington. 
A project funded by U.S. Fleet Forces Command and Naval Engineering Facilities Command Atlantic to measure underwater sound generated by explosions (Soloway and Dahl, 2014) is applying vector acoustic techniques acquired in this project to study Scholte waves.

\section{REFERENCES}

[1] P. H. Dahl, W. J. Plant and D.R. Dall’Osto, Vertical coherence and forward scattering from the sea surface and the relation to the directional wave spectrum, J. Acoust. Soc. Am., 134 (3), 1843-1853, Sept. 2013.

[2] D.R. Dall'Osto and P. H. Dahl, Elliptical acoustic particle motion in underwater waveguides, J. Acoust. Soc. Am., 134 (1), 109-118, July 2013.

\section{PUBLICATIONS}

A. Soloway and P. H. Dahl, Peak sound pressure and sound exposure level from underwater explosions in shallow water, J. Acoust. Soc. Am., Express Letters, 136 (3) 218-223, Sep. 2014. [published, refereed] 